\title{
Nonlinear Oscillator with Power-Form Elastic-Term: Fourier Series Expansion of the Exact Solution
}

\author{
Augusto Beléndez, ${ }^{1,2}$ Jorge Francés, ${ }^{1,2}$ Tarsicio Beléndez, ${ }^{1,2}$ Sergio Bleda, \\ Carolina Pascual, ${ }^{1,2}$ and Enrique Arribas ${ }^{3}$
}

(1) Departamento de Física, Ingeniería de Sistemas y Teoría de la Señal. Universidad de Alicante. Apartado 99. E-03080 Alicante. SPAIN

(2) Instituto Universitario de Física Aplicada a las Ciencias y las Tecnologías. Universidad de Alicante. Apartado 99. E-03080 Alicante. SPAIN

(3) Departamento de Física Aplicada. Universidad de Castilla-La Mancha. Avda. España s/n, E02071 Albacete. SPAIN

\begin{abstract}
A family of conservative, truly nonlinear, oscillators with integer or non-integer order nonlinearity is considered. These oscillators have only one odd power-form elastic-term and exact expressions for their period and solution were found in terms of Gamma functions and a cosine-Ateb function, respectively. Only for a few values of the order of nonlinearity, is it possible to obtain the periodic solution in terms of more common functions. However, for this family of conservative truly nonlinear oscillators we show in this paper that it is possible to obtain the Fourier series expansion of the exact solution, even though this exact solution is unknown. The coefficients of the Fourier series expansion of the exact solution are obtained as an integral expression in which a regularized incomplete Beta function appears. These coefficients are a function of the order of nonlinearity only and are computed numerically. One application of this technique is to compare the amplitudes for the different harmonics of the solution obtained using approximate methods with the exact ones computed numerically as shown in this paper. As an example, the approximate amplitudes obtained via a modified Ritz method are compared with the exact ones computed numerically.
\end{abstract}

Keywords: Dynamical systems; Nonlinear oscillators; Conservative systems; Truly nonlinear oscillators; Fourier series expansion; Approximate solutions; Symbolic computation. 


\section{Introduction}

Nonlinear oscillations in physics, engineering, mathematics and related fields have been the subject of intensive research for many years and several methods have been used to find approximate solutions to these dynamical systems [1,2]. In conservative nonlinear oscillators the restoring force is not dependent on time, the total energy is constant [2,3] and any oscillation is stationary. An important feature of the solutions for conservative oscillators is that they are periodic and range over a continuous interval of initial values [4]. Conservative truly nonlinear oscillatory systems are modelled by differential equations for which the restoring force has no linear approximation at $x=0$ [4].

In this paper we consider a class of conservative truly nonlinear oscillators with an odd power-form elastic-term, $f(x)=\operatorname{sgn}(x)|x|^{\alpha}$, where $\alpha \geq 0$. In recent years some examples of this class of truly nonlinear oscillators have been analyzed. Mickens [5] studied the oscillations in a $x^{4 / 3}$ potential and Hu and Xiong [6] extended this study to a more general $x^{(2 \mathrm{~m}+1)(2 \mathrm{n}+1)}$ potential, where $m$ and $n$ are arbitrary non-negative integers. Several techniques have been used to obtain analytical approximate solutions, such as harmonic balance, parameter expansion, iteration or averaging methods [4-15]. Gottlieb [8] analyzed the frequencies of oscillators with fractional-power nonlinearities introducing the sign function which enables the sign of the restoring force to be changed. He also obtained an expression for the exact period of this type of oscillators. Pilipchuk [16] studied a more general class of these oscillators in which the exponent $\alpha$ of $x$ continuously takes any non-negative real value, such as odd, even, rational or irrational. Pilipchuk obtained an approximate solution as a series expansion and found that this solution is more accurate when the exponent $\alpha$ increases. A new analytical method for solving the differential equations, which describe the motion of the oscillator with fraction order elastic force, was introduced by Cveticanin [14]. In this technique the Krylov-Bogolubov method was extended. By considering a variable frequency, Kovacic and Rakaric [17] developed a procedure to obtain higher-order approximations for oscillators with a fractional-order restoring force. They adjusted the Ritz method by introducing an approximate Lagrangian and using the exact value of the frequency of oscillations they obtained explicit expressions for the amplitudes of the secondand third-order approximations. Cveticanin [18] analyzed the vibrations of oscillators with non-integer order nonlinearity and time variable parameters. Cveticanin and Pogány [19] studied free and self-excited vibrations of oscillators with polynomial nonlinearity. Recently, Elías-Zúñiga and O. Martínez-Romero [20] obtained accurate solutions for a generalized 
power-form elastic term oscillator using the enhanced cubication method developed by these authors. In an interesting paper, Muñoz and Fernández-Anaya [21] showed that this family of conservative truly nonlinear oscillators with an odd power-form elastic-term arose from particular solutions of Abel's mechanical problem [22].

For oscillators in which only one odd power-form elastic-term exists, the exact analytical period can be obtained in terms of Gamma functions using the energy integral [14] and the explicit solution can be expressed as a cosine-Ateb function [19,23], which represents the inversion of the incomplete Beta functions. However, only a few examples of this type of oscillators -such as linear, anti-symmetric constant force, pure-quadratic and pure-cubic oscillators- have closed-form solutions [4]. However, it will be shown in this paper that it is possible to obtain the Fourier series expansion of the exact solution for all of these conservative truly nonlinear oscillators, even though their exact solutions are unknown. The coefficients of the Fourier series expansion of the exact solution are obtained as a function of the order of nonlinearity (the exponent $\alpha$ ) and are computed numerically. Since the nonlinear restoring force is an odd function of the displacement $x$, only the odd harmonics appear in the Fourier series expansion of the periodic solution. We compare the coefficients computed using this method with those obtained for the Fourier series expansion for the exact solution both for the anti-symmetric constant force oscillator and the pure-cubic oscillator. We present some examples to illustrate the usefulness of the technique for obtaining approximate solutions with a finite number of harmonics by truncating the Fourier series expansion. One of the possible applications of this method is to compare the amplitudes for the different harmonics of the solution obtained using approximate methods with the exact ones computed numerically as shown in this paper. As an example, we compare the approximate amplitudes obtained via a modified Ritz method [17] with the exact ones computed numerically. As may be seen, this allows us to determine the range of values for the order of nonlinearity for which the approximate Lagrangian used gives accurate results.

\section{Formulation and solution procedure}

We consider a class of conservative single-degree-of-freedom nonlinear oscillators modelled by the second-order autonomous differential equation

$$
\frac{\mathrm{d}^{2} x}{\mathrm{~d} t^{2}}+\operatorname{sgn}(x)|x|^{\alpha}=0
$$


with initial conditions

$$
x(0)=A, \quad \frac{\mathrm{d} x}{\mathrm{~d} t}(0)=0
$$

where $x$ and $t$ are the non-dimensional displacement and time, respectively, and the order $\alpha$ of nonlinearity is any positive real number $(\alpha \geq 0)$. These oscillators are truly nonlinear oscillators [4] in which the nonlinear function $f(x)=\operatorname{sgn}(x)|x|^{\alpha}$ is odd, i.e. $f(-x)=-f(x)$ and satisfies $x f(x)>0$ for $x \in[-A, A], x \neq 0$, where $A$ is the oscillation amplitude. The system oscillates around the equilibrium position $x=0$ and the period, $T$, and periodic solution, $x$, are dependent on $\alpha$ and $A$.

Introducing a new reduced variable, $u=x / A$, we can rewrite Eqs. (1) and (2) as

$$
\begin{gathered}
\frac{\mathrm{d}^{2} u}{\mathrm{~d} t^{2}}+A^{\alpha-1} \operatorname{sgn}(u)|u|^{\alpha}=0 \\
u(0)=1, \quad \frac{\mathrm{d} u}{\mathrm{~d} t}(0)=0
\end{gathered}
$$

We consider $0 \leq t \leq T / 4$, where $T$ is the period of the oscillations. For these values of $t$ we have $0 \leq u \leq 1$. Integration of Eq. (3) gives the first integral

$$
\frac{1}{2}\left(\frac{d u}{d t}\right)^{2}+\frac{1}{1+\alpha} A^{\alpha-1} u^{1+\alpha}=\frac{1}{1+\alpha} A^{\alpha-1}
$$

where the integration constant was evaluated using the initial conditions of Eq. (4). Solving for $d u / d t$ we obtain

$$
\frac{d u}{d t}=-\sqrt{\frac{2}{1+\alpha}} A^{(\alpha-1) / 2} \sqrt{1-u^{1+\alpha}}
$$

which can be rewritten as

$$
d t=-\sqrt{\frac{1+\alpha}{2}} \frac{A^{(1-\alpha) / 2}}{\sqrt{1-u^{1+\alpha}}} d u
$$

Direct integration of Eq. (7) yields the period $T$ of this family of conservative oscillators as (Appendix I)

$$
T(\alpha, A)=\frac{4 \sqrt{\pi} \Gamma\left(\frac{1}{1+\alpha}\right)}{\sqrt{2(1+\alpha)} \Gamma\left(\frac{3+\alpha}{2+2 \alpha}\right)} A^{(1-\alpha) / 2}
$$


where $\Gamma(z)$ is the Euler gamma function defined as [24]

$$
\Gamma(z)=\int_{0}^{\infty} t^{z-1} \exp (-t) d t
$$

From Eq. (7) we obtain $t$ as a function of $u$ for $0 \leq t \leq T / 4$ as

$$
t(u)=\sqrt{\frac{1+\alpha}{2}} A^{(1-\alpha) / 2} \int_{u}^{1} \frac{d u}{\sqrt{1-u^{1+\alpha}}}, \quad u \geq 0
$$

which can be written as (Appendix II)

$$
t(u)=\frac{\sqrt{\pi} \Gamma\left(\frac{1}{1+\alpha}\right) A^{(1-\alpha) / 2}}{\sqrt{2(1+\alpha)} \Gamma\left(\frac{3+\alpha}{2+2 \alpha}\right)} I\left(1-u^{1+\alpha} ; \frac{1}{2}, \frac{1}{1+\alpha}\right)
$$

In Eq. (10), $I(z ; a, b)$ is the regularized incomplete Beta function [24] defined as

$$
I(z ; a, b) \equiv I_{z}(a, b)=\frac{B(z ; a, b)}{B(a, b)}
$$

where $B(z ; a, b)$ and $B(a, b)$ are the incomplete and complete Beta functions, respectively, defined as follows [24]

$$
\begin{gathered}
B(z ; a, b) \equiv B_{z}(a, b)=\int_{0}^{z} t^{a-1}(1-t)^{b-1} d t \\
B(a, b)=\int_{0}^{1} t^{a-1}(1-t)^{b-1} d t
\end{gathered}
$$

Since the nonlinear function in Eq. (3) is an odd function of $u$, the periodic solution $u$ can be expanded in a Fourier series as

$$
u(\alpha, t)=\sum_{n=0}^{\infty} a_{2 n+1}(\alpha) \cos \left[(2 n+1) \frac{2 \pi t}{T(\alpha, A)}\right]
$$

where the coefficients $a_{2 n+1}$ are given by

$$
a_{2 n+1}(\alpha)=\frac{8}{T} \int_{0}^{T / 4} u(\alpha, t) \cos \left[(2 n+1) \frac{2 \pi t}{T(\alpha, A)}\right] d t
$$

taking into account that the periodic solution $u$ does not depend on the oscillation amplitude $A$. Substituting Eqs. (8) and (11) into Eq. (16) gives

$$
a_{2 n+1}(\alpha)=\frac{2(1+\alpha) \Gamma\left(\frac{3+\alpha}{2+2 \alpha}\right)}{\sqrt{\pi} \Gamma\left(\frac{1}{1+\alpha}\right)} \int_{0}^{1} \frac{u}{\sqrt{1-u^{1+\alpha}}} \cos \left[\frac{(2 n+1) \pi}{2} I\left(1-u^{1+\alpha} ; \frac{1}{2}, \frac{1}{1+\alpha}\right)\right] d u
$$


Let $y=1-u^{1+\alpha}$, then Eq. (17) becomes

$$
a_{2 n+1}(\alpha)=\frac{2 \Gamma\left(\frac{3+\alpha}{2+2 \alpha}\right)}{\sqrt{\pi} \Gamma\left(\frac{1}{1+\alpha}\right)} \int_{0}^{(1-y)^{(1-\alpha) /(1+\alpha)}} \cos \left[\frac{(2 n+1) \pi}{2} I\left(y ; \frac{1}{2}, \frac{1}{1+\alpha}\right)\right] d y
$$

This equation allows us to obtain the values of the coefficients $a_{2 n+1}(\alpha)$ of the Fourier series expansion of the periodic solution $u(\alpha, t)$ as a function of the order of nonlinearity $\alpha$, even though the analytical expression of this solution is unknown. In general, the integral in Eq. (18) must be computed numerically and this was done with the help of symbolic computation software such as MATHEMATICA.

The first four Fourier coefficients were computed as a function of the order of nonlinearity $\alpha$ using Eq. (18). In Figure 1 we plotted the normalized first Fourier coefficient, $a_{1}$, as a function of the order of nonlinearity, $\alpha$, for $0 \leq \alpha \leq 12$. From this figure it may be seen that $a_{1}>1$ for $0 \leq \alpha<1$ ( $a_{1} \approx 1.032$ for $\alpha=0$, anti-symmetric constant force oscillator), $a_{1}=1$ for $\alpha=1$ (linear oscillator) and $a_{1}<1$ for $\alpha>1$. This coefficient decreases when the order of nonlinearity increases. Figure 2 shows the normalized second Fourier coefficient, $a_{3}$, as a function of the order of nonlinearity, $\alpha$. In this case $a_{3}<0$ for $0 \leq \alpha<1\left(a_{3} \approx-0.038\right.$ for $\alpha=$ 0 , anti-symmetric constant force oscillator), $a_{3}=1$ for $\alpha=1$ (linear oscillator) and $a_{3}>0$ for $\alpha>1$, and this coefficient is seen to increase when the order of nonlinearity increases. In Figure 3 we plotted the third Fourier coefficient, $a_{5}$, as a function of the order of nonlinearity, $\alpha$, and we can see that $a_{5}>0$ for $0 \leq \alpha<1$ and for $\alpha>2.34$, and $a_{5}<0$ for $1 \leq$ $\alpha<2.34$. The coefficient $a_{5}$ decreases when $\alpha$ increases from 0 to 1.60 and for values of $\alpha$ higher than 1.60, $a_{5}$ increases when $\alpha$ increases. It is important to point out that $a_{5}$ is equal to zero not only for $\alpha=1$, but also for $\alpha \approx 2.34$. Finally, Figure 4 shows the behaviour of the fourth Fourier coefficient, $a_{7}$. In this case, $a_{7}<0$ for $0 \leq \alpha<1$ and $a_{7} \geq 0$ for $\alpha \geq 1$. The coefficient $a_{7}$ increases from $\alpha=0$ to $\alpha \approx 1.55$, decreases from $\alpha \approx 1.55$ to $\alpha \approx 2.72$, and increases again from $\alpha \approx 2.72$.

\section{Some nonlinear oscillators with analytical exact solutions}

In this section we consider two values of parameter $\alpha$ for which exact analytical solutions exist. Then it is possible to do the Fourier series expansions of these solutions in order to 
obtain the values of the coefficients $a_{2 n+1}$ in Eq. (16) and these can be compared with those obtained using Eq. (18).

Anti-symmetric, constant force oscillator $(\alpha=0)$ : The nonlinear differential equation for the anti-symmetric, piecewise constant force oscillator is

$$
\frac{\mathrm{d}^{2} u}{\mathrm{~d} t^{2}}+\frac{1}{A} \operatorname{sgn}(u)=0
$$

For this nonlinear problem, the exact $T$ and periodic solution $u$ are $[2,10]$

$$
\begin{gathered}
T \equiv T(0, A)=4 \sqrt{2 A} \\
u(t) \equiv u(0, t)=\left\{\begin{array}{cc}
-\frac{t^{2}}{2 A}+1, & 0 \leq t \leq \frac{T}{4} \\
\frac{t^{2}}{2 A}-2 \sqrt{\frac{2}{A}} t+3, & \frac{T}{4} \leq t \leq \frac{3 T}{4} \\
-\frac{t^{2}}{2 A}+4 \sqrt{\frac{2}{A}} t-15, & \frac{3 T}{4} \leq t \leq T
\end{array}\right.
\end{gathered}
$$

A simple and direct calculation gives the following series representation for the exact solution in Eq. (21)

$$
u(0, t)=\frac{32}{\pi^{3}} \sum_{n=0}^{\infty} \frac{(-1)^{n}}{(2 n+1)^{3}} \cos \left[(2 n+1) \frac{2 \pi t}{T}\right]
$$

and the Fourier coefficients are given as follows [10]

$$
a_{2 n+1}(0)=\frac{(-1)^{n}}{(2 n+1)^{3}} \frac{32}{\pi^{3}}
$$

For $\alpha=0$, Eq. (18) gives

$$
a_{2 n+1}(0)=\int_{0}^{1} \frac{-y}{\sqrt{y}} \cos \left[(2 n+1) \frac{\pi}{2} \sqrt{y}\right] d y=\frac{(-1)^{n}}{(2 n+1)^{3}} \frac{32}{\pi^{3}}
$$

As we can see, the Fourier coefficients obtained using Eq. (18) coincide with the coefficients in Eq. (23) obtained from the Fourier series expansion of the exact solution in Eq. (21). The first of these coefficients are

$$
a_{1}(0)=\frac{32}{\pi^{3}} \approx 1.03205, \quad a_{3}(0)=-\frac{32}{27 \pi^{3}} \approx-0.038004, \quad a_{5}(0)=\frac{32}{125 \pi^{3}} \approx 0.00825639
$$


Pure-cubic oscillator $(\alpha=3)$ : This oscillator is governed by the following differential equation

$$
\frac{\mathrm{d}^{2} u}{\mathrm{~d} t^{2}}+A^{2} u^{3}=0
$$

For this oscillator, the exact $T$ and periodic solution $u$ are [2]

$$
\begin{aligned}
& T \equiv T(3, A)=\frac{4 K(1 / 2)}{A} \\
& u(t) \equiv u(3, t)=\operatorname{cn}\left(A t ; \frac{1}{2}\right)
\end{aligned}
$$

where $K(m)$ is the complete elliptic integral of the first kind defined as follows [25]

$$
K(m)=\int_{0}^{\pi / 2} \frac{\mathrm{d} \theta}{\sqrt{1-m \sin ^{2} \theta}}
$$

and $\operatorname{cn}(z ; m)$ is the Jacobi elliptic function which has the following expansion in Fourier series [26]

$$
\operatorname{cn}(z ; m)=\frac{2 \pi}{\sqrt{m} K(m)} \sum_{n=0}^{\infty} \frac{q^{n+1 / 2}}{1+q^{2 n+1}} \cos \left[\frac{(2 n+1) \pi z}{2 K(m)}\right]
$$

where

$$
q(m)=\exp \left[-\frac{\pi K\left(m^{\prime}\right)}{K(m)}\right]
$$

and $m^{\prime}=1-m$. With these results, the Fourier series expansion of Eq. (28) becomes $[2,11]$

$$
u(3, t)=\operatorname{cn}\left(A t ; \frac{1}{2}\right)=\frac{2 \pi \sqrt{2}}{K(1 / 2)} \sum_{n=0}^{\infty} \frac{\exp [(n+1 / 2) \pi]}{1+\exp [(2 n+1) \pi]} \cos \left[(2 n+1) \frac{2 \pi t}{T}\right]
$$

and the Fourier coefficients are given as follows

$$
a_{2 n+1}(3)=\frac{2 \pi \sqrt{2}}{K(1 / 2)}\left(\frac{\exp [(n+1 / 2) \pi]}{1+\exp [(2 n+1) \pi]}\right)
$$

For $\alpha=3$, Eq. (18) gives

$$
a_{2 n+1}(3)=\frac{2 \Gamma(3 / 4)}{\sqrt{\pi} \Gamma(1 / 4)} \int_{0}^{1} \frac{1}{\sqrt{y(1-y)}} \cos \left[\frac{(2 n+1) \pi}{2} I\left(y ; \frac{1}{2}, \frac{1}{4}\right)\right] d y
$$


In Table 1 we present the values of the first five Fourier coefficients calculated using Eqs. (33) and (34) as well as the relative error of the coefficients computed using Eq. (33). As we can see, the agreement is excellent.

\section{Analytical approximate solutions}

In this section, we present some examples to illustrate the usefulness of the technique for obtaining the coefficients of Fourier expansion of the periodic solution. As the exact solution has an infinite number of harmonics (see Eq. (15)), we truncate the series expansion at Eq. (15) and we consider an analytical approximate solution of the form

$$
u^{(N)}(\alpha, t)=a_{1}^{(N)}(\alpha) \cos \left[\frac{2 \pi t}{T(\alpha, A)}\right]+\sum_{n=1}^{N} a_{2 n+1}(\alpha) \cos \left[(2 n+1) \frac{2 \pi t}{T(\alpha, A)}\right]
$$

where the coefficient of the first harmonic, $a_{1}^{(N)}$, is obtained by using the first of the initial conditions in Eq. (4)

$$
a_{1}^{(N)}(\alpha)=1-\sum_{n=1}^{N} a_{2 n+1}(\alpha)
$$

In Tables 2-7 we present the values of $a_{1}^{(N)}$ for different values of $\alpha$ and $N$, as well as the exact values of $a_{1}$. Some common oscillators were considered and the approximate values of $a_{1}^{(N)}$ and their relative errors were calculated. In Figures 5-10 the approximate solution (dashed line and triangles) is compared with the numerical exact solution (continuous line and squares) for the oscillators considered in Tables 2-7 ( $\alpha=0,1 / 3,2,3,5$ and 12). For small nonlinearities the Fourier coefficients decrease rapidly and, consequently, the use of just a few Fourier coefficients provides an accurate representation of the exact periodic solution [4]. However, for large values of the order of nonlinearity (exponent $\alpha$ ), it is necessary to take into account more coefficients in the Fourier series expansion in order to obtain accurate results. 


\section{Comparison with the approximate solutions obtained using a modified Ritz method}

Kovacic and Rakaric [17] adjusted the Ritz method to obtain higher-order approximations for motion of the conservative oscillatory systems modelled by Eq. (1). In their modified Ritz method, the exact period is used and an approximation of the Lagrangian is introduced [17, page. 2653, Eqs. (10a,b) and (11)]. They expressed both the kinetic and the potential energy as series expansions and truncated these series considering only the first three terms in each of these expansions. Using this procedure, they obtained explicit equations for the amplitudes of the second and third approximations for motion, which may be written as follows

$$
\begin{gathered}
u^{(1)}(\alpha, t)=b_{1}(\alpha) \cos \left[\frac{2 \pi t}{T(\alpha, A)}\right]+b_{3}(\alpha) \cos \left[\frac{6 \pi t}{T(\alpha, A)}\right] \\
u^{(2)}(\alpha, t)=c_{1}(\alpha) \cos \left[\frac{2 \pi t}{T(\alpha, A)}\right]+c_{3}(\alpha) \cos \left[\frac{6 \pi t}{T(\alpha, A)}\right]+c_{5}(\alpha) \cos \left[\frac{10 \pi t}{T(\alpha, A)}\right]
\end{gathered}
$$

the amplitudes of which satisfy the initial conditions in Eq. (4)

$$
b_{1}(\alpha)=1-b_{3}(\alpha) \quad \text { and } \quad c_{1}(\alpha)=1-c_{3}(\alpha)-c_{5}(\alpha)
$$

Applying the modified Ritz method proposed by Kovacic and Rakaric, we obtained the expressions for these amplitudes, which are included the Appendix III. As can be seen, the amplitudes we have obtained are valid for $\alpha \geq 0$, whereas those obtained by Kovacic and Rakaric can be only used for $\alpha \geq 1$. The first four exact Fourier coefficients as well as those obtained using the second, third and fourth approximations for motion applying the modified Ritz method are plotted in Figures 11-14. These coefficients are plotted as a function of the order of nonlinearity, $\alpha$. We also obtained the fourth approximation, which was not calculated in reference [17], but its expressions are not included in this paper. As can be seen in Figures 11 and 12, the amplitudes of the second approximation coincide with the exact values of the Fourier coefficients only up to $\alpha \approx 3$, and this implies that the second approximation can be used in this region. The third approximation is obviously better than the second one. The first and the second approximate Fourier coefficients are better than those obtained using the second approximation. As can be seen in Figures 11-13, this approximation gives accurate values for the Fourier coefficient only up to $\alpha \approx 4$. Finally, from Figures 11-14 we conclude that the results obtained using the fourth approximation are 
not much better than those calculated using the third approximation, with accurate values for the Fourier coefficients up to $\alpha \approx 4$. This means that using higher approximations does not improve the results for $\alpha \geq 4$ and when the order of nonlinearity, $\alpha$, increases, it is necessary to consider more than three terms in the potential energy series expansion [17, page. 2653, Eq. $(10 a, b)]$.

\section{Conclusions}

The coefficients of the Fourier series expansion of the exact solution for a family of conservative, truly nonlinear, oscillators with integer or non-integer order nonlinearity were calculated. They were expressed as an integral in which a regularized incomplete Beta function appears and they depend on the order of nonlinearity. These integrals were computed numerically and their results plotted as a function of the order of nonlinearity. The Fourier series expansion of the exact solutions of the anti-symmetric constant force, and pure-cubic oscillators were compared with the Fourier series expansions obtained using the procedure proposed in this paper. By truncating the Fourier series expansion approximate solutions with a finite number of harmonics have been obtained and these solutions were compared with the exact ones. We also showed that finding the Fourier series expansions of the exact solution -even though this solution is not known- allows us to analyze the accuracy of analytical approximate methods. To do this, we compared the Fourier series expansions of the approximate solutions obtained using a modified Ritz method with the exact ones and found that this approximate method gives accurate solutions for values of an order of nonlinearity less than 3. Finally, this procedure can be applied to other types of conservative nonlinear oscillators -without analytical expressions for their exact solutionsand in each case it would be possible to determine the number of Fourier coefficients necessary to provide an accurate approximate representation of the exact periodic solution.

\section{Appendix I}

We consider $0 \leq t \leq T / 4$, where $T$ is the period of the oscillations. For these values of $t$ we have $0 \leq u \leq 1$. Integration of Eq. (3) gives the first integral

$$
\frac{1}{2}\left(\frac{d u}{d t}\right)^{2}+\frac{1}{1+\alpha} A^{\alpha-1} u^{1+\alpha}=\frac{1}{1+\alpha} A^{\alpha-1}
$$


where the constant of integration was evaluated using the initial boundary conditions in Eq. (4). Solving for $d u / d t$ gives

$$
\frac{d u}{d t}=-\sqrt{\frac{2}{1+\alpha}} A^{(\alpha-1) / 2} \sqrt{1-u^{1+\alpha}}
$$

This equation can be rewritten as

$$
d t=-\sqrt{\frac{1+\alpha}{2}} \frac{A^{(1-\alpha) / 2}}{\sqrt{1-u^{1+\alpha}}} d u
$$

The time it takes to go from point $(A, 0)$ to the point $(u, d u / d t)$ in the lower half-plane of the phase space is

$$
t(u)=-\sqrt{\frac{1+\alpha}{2}} A^{(1-\alpha) / 2} \int_{1}^{u} \frac{d u}{\sqrt{1-u^{1+\alpha}}}=\sqrt{\frac{1+\alpha}{2}} A^{(1-\alpha) / 2} \int_{u}^{1}\left(1-u^{1+\alpha}\right)^{-1 / 2} d u
$$

The period of oscillation is four times the time taken by the oscillator to go from $u=0$ to $u=$ $1, T=4 t(0)$. Therefore

$$
T=2 \sqrt{2(1+\alpha)} A^{(1-\alpha) / 2} \int_{0}^{1}\left(1-u^{1+\alpha}\right)^{-1 / 2} d u
$$

The substitution $z=u^{1+\alpha}$ gives, after some simplifications

$$
T=2 \sqrt{\frac{2}{1+\alpha}} A^{(1-\alpha) / 2} \int_{0}^{1} z^{-\alpha /(1+\alpha)}(1-z)^{-1 / 2} d z=2 \sqrt{\frac{2}{1+\alpha}} A^{(1-\alpha) / 2} B\left(\frac{1}{1+\alpha}, \frac{1}{2}\right)
$$

where $B(a, b)$ is the Beta function. Finally, Eq. (A6) can be rewritten in terms of Gamma functions as follows

$$
T(\alpha, A)=\frac{4 \sqrt{\pi} \Gamma\left(\frac{1}{1+\alpha}\right) A^{(1-\alpha) / 2}}{\sqrt{2(1+\alpha)} \Gamma\left(\frac{3+\alpha}{2(1+\alpha)}\right)}
$$

\section{Appendix II}

Substituting $z=u^{1+\alpha}$ in Eq. (A4) and following the method used by MacColl for the relativistic oscillator [27] and by Méndez et al to find approximate solutions for nonlinear Hamiltonian oscillators [28], we obtain, after some simplifications 


$$
t(z)=\frac{1}{\sqrt{2(1+\alpha)}} A^{(1-\alpha) / 2} \int_{z}^{1} z^{-\alpha /(1+\alpha)}(1-z)^{-1 / 2} d z
$$

which can be written as

$$
t(z)=\frac{1}{\sqrt{2(1+\alpha)}} A^{(1-\alpha) / 2} \int_{0}^{1} z^{-\alpha /(1+\alpha)}(1-z)^{-1 / 2} d z-\frac{1}{\sqrt{2(1+\alpha)}} A^{(1-\alpha) / 2} \int_{0}^{z} z^{-\alpha /(1+\alpha)}(1-z)^{-1 / 2} d z \text { (A9) }
$$

whose value is

$$
t(u)=\frac{A^{(1-\alpha) / 2}}{\sqrt{2(1+\alpha)}}\left[\frac{\sqrt{\pi} \Gamma\left(\frac{1}{1+\alpha}\right)}{\Gamma\left(\frac{3+\alpha}{2(1+\alpha)}\right)}-B\left(u^{1+\alpha} ; \frac{1}{1+\alpha}, \frac{1}{2}\right)\right]
$$

where $B(\mathrm{z} ; a, b)$ is the incomplete Beta function. Eq. (A10) can be written in terms of the regularized incomplete Beta function, $I(z ; a, b)$, as follows

$$
t(u)=\frac{\sqrt{\pi} \Gamma\left(\frac{1}{1+\alpha}\right) A^{(1-\alpha) / 2}}{\sqrt{2(1+\alpha)} \Gamma\left(\frac{3+\alpha}{2+2 \alpha}\right)}\left[1-I\left(u^{1+\alpha} ; \frac{1}{1+\alpha}, \frac{1}{2}\right)\right]
$$

and taking into account that

$$
I(z ; a, b)=1-I(1-z ; b, a)
$$

we can write Eq. (A11) as

$$
t(u)=\frac{\sqrt{\pi} \Gamma\left(\frac{1}{1+\alpha}\right) A^{(1-\alpha) / 2}}{\sqrt{2(1+\alpha)} \Gamma\left(\frac{3+\alpha}{2+2 \alpha}\right)} I\left(1-u^{1+\alpha} ; \frac{1}{2}, \frac{1}{1+\alpha}\right)
$$

\section{Appendix III}

Expression for the amplitudes corresponding to the second-order approximation obtained using the modified Ritz method proposed by Kovacic and Rakaric [17]

$$
\begin{gathered}
b_{1}(\alpha)=1-b_{3}(\alpha) \\
b_{3}(\alpha)=\frac{(\alpha-1)(\alpha+5)(\alpha+7)(\alpha+9) \Gamma\left(1+\frac{\alpha}{2}\right) \Gamma^{2}\left(\frac{1}{1+\alpha}\right)}{4 \alpha(\alpha-7)(\alpha+7)(\alpha+9) \Gamma\left(1+\frac{\alpha}{2}\right) \Gamma^{2}\left(\frac{1}{1+\alpha}\right)+36 \pi^{3 / 2}(\alpha+1) \Gamma\left(\frac{11+\alpha}{2}\right) \Gamma^{2}\left(\frac{3+\alpha}{2+2 \alpha}\right)}
\end{gathered}
$$

and for the third-order approximation we obtain

$$
\begin{gathered}
c_{1}(\alpha)=1-c_{3}(\alpha)-c_{5}(\alpha) \\
c_{3}(\alpha)=\frac{p_{1}(\alpha)\left[p_{2}(\alpha)-p_{3}(\alpha)\right]}{q_{1}(\alpha)+q_{2}(\alpha)+q_{3}(\alpha)}
\end{gathered}
$$




$$
c_{5}(\alpha)=\frac{r_{1}(\alpha)\left[r_{2}(\alpha)+r_{3}(\alpha)\right]}{s_{1}(\alpha)+s_{2}(\alpha)+s_{3}(\alpha)}
$$

where

$$
\begin{aligned}
& p_{1}(\alpha)=-(\alpha-1)(\alpha+7)(\alpha+9) \Gamma\left(1+\frac{\alpha}{2}\right) \Gamma^{2}\left(\frac{1}{1+\alpha}\right) \\
& p_{2}(\alpha)=96 \alpha\left(17+2 \alpha+\alpha^{2}\right) \Gamma\left(2+\frac{\alpha}{2}\right) \Gamma^{2}\left(\frac{1}{1+\alpha}\right) \\
& p_{3}(\alpha)=25 \pi^{3 / 2}(\alpha+1)(\alpha+5) \Gamma\left(\frac{11+\alpha}{2}\right) \Gamma^{2}\left(\frac{3+\alpha}{2+2 \alpha}\right) \\
& q_{1}(\alpha)=768 \alpha^{2}(\alpha+2)(\alpha+9)\left(43-4 \alpha+\alpha^{2}\right) \Gamma^{2}\left(1+\frac{\alpha}{2}\right) \Gamma^{4}\left(\frac{1}{1+\alpha}\right) \\
& q_{2}(\alpha)=16 \pi^{3 / 2}\left(-3600-4021 \alpha-493 \alpha^{2}-59 \alpha^{3}+13 \alpha^{4}\right) \Gamma\left(1+\frac{\alpha}{2}\right) \Gamma^{2}\left(\frac{1}{1+\alpha}\right) \Gamma\left(\frac{11+\alpha}{2}\right) \Gamma^{2}\left(\frac{3+\alpha}{2+2 \alpha}\right) \\
& q_{3}(\alpha)=900 \pi^{3}(\alpha+1)^{2} \Gamma^{2}\left(\frac{11+\alpha}{2}\right) \Gamma^{4}\left(\frac{3+\alpha}{2+2 \alpha}\right) \\
& r_{1}(\alpha)=\left(63-16 \alpha+62 \alpha^{2}+16 \alpha^{3}+\alpha^{4}\right) \Gamma\left(1+\frac{\alpha}{2}\right) \Gamma^{2}\left(\frac{1}{1+\alpha}\right) \\
& r_{2}(\alpha)=32 \alpha(\alpha+9)\left(2+\frac{\alpha}{2}\right) \Gamma^{2}\left(\frac{1}{1+\alpha}\right) \\
& r_{3}(\alpha)=9 \pi^{3 / 2}(\alpha-3) \Gamma\left(\frac{11+\alpha}{2}\right) \Gamma^{2}\left(\frac{3+\alpha}{2+2 \alpha}\right) \\
& s_{1}(\alpha)=768 \alpha^{2}\left(774+401 \alpha+17 \alpha^{2}+7 \alpha^{3}+\alpha^{4}\right) \Gamma^{2}\left(1+\frac{\alpha}{2}\right) \Gamma^{4}\left(\frac{1}{1+\alpha}\right) \\
& s_{2}(\alpha)=16 \pi^{3 / 2} \alpha\left(-3600-4021 \alpha-493 \alpha^{2}-59 \alpha^{3}+13 \alpha^{4}\right) \Gamma\left(1+\frac{\alpha}{2}\right) \Gamma^{2}\left(\frac{1}{1+\alpha}\right) \Gamma\left(\frac{11+\alpha}{2}\right) \Gamma^{2}\left(\frac{3+\alpha}{2+2 \alpha}\right) \\
& s_{3}(\alpha)=900 \pi^{3}(\alpha+1)^{2} \Gamma^{2}\left(\frac{11+\alpha}{2}\right) \Gamma^{4}\left(\frac{3+\alpha}{2+2 \alpha}\right) \\
&
\end{aligned}
$$

\section{Acknowledgements}

This work was supported by the "Generalitat Valenciana" of Spain, under projects PROMETEO/2011/021 and ISIC/2012/013, and by the "Vicerrectorado de Tecnologías de la Información" of the University of Alicante, Spain, under project GITE-09006-UA. 


\section{References}

[1] A. H. Nayfeh, Problems in Perturbation, John Wiley \& Sons, New York, 1985.

[2] R. E. Mickens, Oscillations in Planar Dynamics Systems, World Scientific, Singapore, 1996.

[3] A. H. Nayfeh and D. T. Mook, Nonlinear Oscillations, Wiley-Interscience, New York, 1979.

[4] R. E. Mickens, Truly Nonlinear Oscillations, World Scientific, Singapore, 2010.

[5] R. E. Mickens, "Oscillations in an $x^{4 / 3}$ potential," Journal of Sound and Vibration, vol. 246, no. 2, pp. 375-378, 2001.

[6] H. Hu and Z.-G. Xiong, "Oscillations in an $x^{(2 m+2) /(2 n+1)}$ potential," Journal of Sound and Vibration, vol. 259, no. 4, pp. 977-980, 2003.

[7] K. Cooper and R. E. Mickens, "Generalized harmonic balance/numerical method for determining analytical approximations to the periodic solutions of the $x^{4 / 3}$ potential," Journal of Sound and Vibration, vol. 250, no. 5, pp. 951-954, 2002.

[8] H. P. W. Gottlieb, "Frequencies of oscillators with fractional-power non-linearities," Journal of Sound and Vibration, vol. 261, no. 3, pp. 557-566, 2003.

[9] A. Beléndez, E. Gimeno, M. L. Alvarez, M. S. Yebra and D. I. Méndez, “Analytical approximate solutions for conservative nonlinear oscillators by modified rational harmonic balance method," International Journal of Computer Mathematics, vol. 87, no. 7, pp. 1497-1511, 2010.

[10] A. Beléndez, A. Hernández, T. Beléndez, C. Neipp and A. Márquez, "Higher accuracy analytical approximations to a nonlinear oscillator with discontinuity by He's homotopy perturbation method," Physics Letters A, vol. 372, no. 12, pp. 2010-2016, 2008.

[11] A. Beléndez, T. Beléndez, A. Márquez and C. Neipp, “Application of He’s homotopy perturbation method to conservative truly nonlinear oscillators," Chaos, Solitons \& Fractals, vol. 37, no. 3, pp. 770-780, 2008. 
[12] V. Marinca and N. Herişanu, "Approximate analytic solutions for non-linear oscillators with fractional-order restoring force by means of the optimal variational iteration method," International Journal of Physical Sciences, vol. 7, no. 37, pp. 5407-5414, 2012.

[13] R. E. Mickens, "Iteration method solutions for conservative and limit-cycle $x^{1 / 3}$ force oscillators," Journal of Sound and Vibration, vol. 292, no. 3-5, pp. 964-968, 2006.

[14] L. Cveticanin, "Oscillator with fraction order restoring force,” Journal of Sound and Vibration, vol. 320, no. 4-5, pp. 1064-1077, 2009.

[15] I. Kovacic, Z. Rakaric, and L. Cveticanin, "A non-simultaneous variational approach for the oscillators with fractional-order power nonlinearities," Applied Mathematics and Computation, vol. 217, no. 8, pp. 3944-3954, 2010.

[16] V. N. Pilipchuk, "Oscillators with a generalized power-form elastic term," Journal of Sound and Vibration, vol. 270, no. 4-5, pp. 470-472, 2004.

[17] I. Kovacic and Z. Rakaric, "Oscillators with a fractional-order restoring force: Higherorder approximations for motion via a modified Ritz method," Communications in Nonlinear Science and Numerical Simulation, vol. 15, no. 9, pp. 2651-2658, 2010.

[18] L. Cveticanin, "Oscillator with non-integer order nonlinearity and time variable parameters," Acta Mechanica, vol. 223, no. 7, pp. 1417-1429, 2012.

[19] L. Cveticanin and T. Pogány, "Oscillator with a sum of noninteger-order nonlinearities," Journal of Applied Mathematics, vol. 2012, Art. ID 649050, 20 pages 2012.

[20] A. Elías-Zúñiga and O. Martínez-Romero, "Accurate Solutions of Conservative Nonlinear Oscillators by the Enhanced Cubication Method," Mathematical Problems in Engineering, vol. 2013, Article ID 842423, 9 pages, 2013.

[21] R. Muñoz and G. Fernández-Anaya, "Conservative nonlinear oscillators in Abel's mechanical problem," Revista Mexicana de Física E, vol. 57, no. 1, pp. 67-72, 2011.

[22] G. F. Simmons and J. S. Robertson, Differential Equations with Applications and Historial Notes, Mc Graw-Hill, New York, 1972. 
[23] R. M. Rosenberg, "The ateb(h)-functions and their properties," Quarterly of Applied Mathematics, vol. 21, pp. 37-47, 1963.

[24] P. J. Davis, "Gamma Function and Related Functions", Ch. 6 in Handbook of Mathematical functions with Formulas, Graphics and Mathematical Tables. M. Abramowitz and I. A. Stegun (Eds.). New York, Dover, pp. 253-266, 1972.

[25] L. M. Milne-Thomson, "Elliptic integrals", Ch. 17 in Handbook of Mathematical functions with Formulas, Graphics and Mathematical Tables. M. Abramowitz and I. A. Stegun (Eds.). New York, Dover, pp. 587-607, 1972.

[26] L. M. Milne-Thomson, "Jacobian elliptic functions and theta functions", Ch. 16 in Handbook of Mathematical functions with Formulas, Graphics and Mathematical Tables. M. Abramowitz and I. A. Stegun (Eds.). New York, Dover, pp. 567-581, 1972.

[27] L. A. MacColl, "Theory of the Relativistic Oscillator," American Journal of Physics, vol. 25, no. 8, pp. 535-538, 1957.

[28] V. Méndez, C. Sanz, D. Campos and I. Llopis, "Fourier series expansion for nonlinear Hamiltonian oscillators,” Physical Review E, vol. 81, Article ID 066201, 8 pages, 2010. 


\section{FIGURE CAPTIONS}

Figure 1.- First Fourier coefficient, $a_{1}$, as a function of the order of nonlinearity (exponent $\alpha$ ) for $0 \leq \alpha \leq 12$.

Figure 2.- Second Fourier coefficient, $a_{3}$, as a function of the order of nonlinearity (exponent $\alpha$ ) for $0 \leq \alpha \leq 12$.

Figure 3.- Third Fourier coefficient, $a_{5}$, as a function of the order of nonlinearity (exponent $\alpha$ ) for $0 \leq \alpha \leq 12$.

Figure 4.- Fourth Fourier coefficient, $a_{7}$, as a function of the order of nonlinearity (exponent $\alpha$ ) for $0 \leq \alpha \leq 12$.

Figure 5. Comparison of the approximate solution (dashed line and triangles) with the numerical solution (continuous line and squares) for $\alpha=0$ and $N=2$ (three harmonics). $a_{1}^{(2)}$ $=1.03205, a_{3}=-0.03822404$ and $a_{5}=0.008256393$.

Figure 6. Comparison of the approximate solution (dashed line and triangles) with the numerical solution (continuous line and squares) for $\alpha=1 / 3$ and $N=2$ (three harmonics). $a_{1}^{(2)}=1.01957, a_{3}=-0.023843$ and $a_{5}=0.00427545$.

Figure 7. Comparison of the approximate solution (dashed line and triangles) with the numerical solution (continuous line and squares) for $\alpha=2$ and $N=1$ (two harmonics). $a_{1}^{(1)}=$ 0.974276 and $a_{3}=0.0257238$.

Figure 8. Comparison of the approximate solution (dashed line and triangles) with the numerical solution (continuous line and squares) for $\alpha=3$ and $N=2$ (three harmonics). $a_{1}^{(2)}$ $=0.95509, a_{3}=0.0430495$ and $a_{5}=0.00186049$.

Figure 9. Comparison of the approximate solution (dashed line and triangles) with the numerical solution (continuous line and squares) for $\alpha=5$ and $N=3$ (four harmonics). $a_{1}^{(3)}$ $=0.926632, a_{3}=0.0633949, a_{5}=0.00877599$ and $a_{7}=0.00119669$. 
Figure 10. Comparison of the approximate solution (dashed line and triangles) with the numerical solution (continuous line and squares) for $\alpha=12$ and $N=5$ (six harmonics). $a_{1}^{(5)}$ $=0.879193, a_{3}=0.0849494, a_{5}=0.0233867, a_{7}=0.00816089, a_{9}=0.00309246$ and $a_{11}=$ 0.00121723 .

Figure 11. First Fourier coefficient as a function of the order of nonlinearity (exponent $\alpha$ ): Exact (black continuous line). Second- (blue dashed line, squares), third- (red dashed line, circles) and fourth- (green dashed line, triangles) approximations obtained applying the modified Ritz method [17].

Figure 12. Second Fourier coefficient as a function of the order of nonlinearity (exponent $\alpha$ ): Exact (black continuous line). Second- (blue dashed line, squares), third- (red dashed line, circles) and fourth- (green dashed line, triangles) approximations obtained applying the modified Ritz method [17].

Figure 13. Third Fourier coefficient as a function of the order of nonlinearity (exponent $\alpha$ ): Exact (black continuous line). Third- (red dashed line, circles) and fourth- (green dashed line, triangles) approximations obtained applying the modified Ritz method [17].

Figure 14. Fourth Fourier coefficient as a function of the order of nonlinearity (exponent $\alpha$ ): Exact (black continuous line). Fourth-approximation (green dashed line) obtained applying the modified Ritz method [17]. 


\section{TABLES}

Table 1. The values of the first five Fourier coefficients for $\alpha=3$ calculated using Eqs. (33) and (34), as well as the relative error of the coefficients computed using Eq. (33).

Table 2. Approximate first Fourier coefficient, $a_{1}^{(N)}$, for different values of $N$ and relative errors for $\alpha=0$. The exact value is $a_{1}=1.03205$.

Table 3. Approximate first Fourier coefficient, $a_{1}^{(N)}$, for different values of $N$ and relative errors for $\alpha=1 / 3$. The exact value is $a_{1}=1.02055$.

Table 4. Approximate first Fourier coefficient, $a_{1}^{(N)}$, for different values of $N$ and relative errors for $\alpha=2$. The exact value is $a_{1}=0.974801$.

Table 5. Approximate first Fourier coefficient, $a_{1}^{(N)}$, for different values of $N$ and relative errors for $\alpha=3$. The exact value is $a_{1}=0.955006$.

Table 6. Approximate first Fourier coefficient, $a_{1}^{(N)}$, for different values of $N$ and relative errors for $\alpha=5$. The exact value is $a_{1}=0.926430$.

Table 7. Approximate first Fourier coefficient, $a_{1}^{(N)}$, for different values of $N$ and relative errors for $\alpha=12$. The exact value is $a_{1}=0.878356$. 


\section{TABLE 1}

\begin{tabular}{cccc}
\hline $\begin{array}{c}\text { Fourier } \\
\text { coefficient }\end{array}$ & Eq. (33) & Eq. (34) & $\begin{array}{c}\text { Relative } \\
\text { error (\%) }\end{array}$ \\
\hline$a_{1}$ & 0.9550059869606213 & 0.9550059869606203 & $10^{-13}$ \\
$a_{3}$ & 0.0430494955774923 & 0.0430494955774919 & $10^{-12}$ \\
$a_{5}$ & 0.00186048723113327 & 0.00186048723113306 & $10^{-11}$ \\
$a_{7}$ & 0.00008039895523055 & 0.00008039895523061 & $7 \times 10^{-11}$ \\
$a_{9}$ & 0.0000034743538810 & 0.0000034743538808 & $7 \times 10^{-9}$ \\
\hline
\end{tabular}


TABLE 2

\begin{tabular}{ccc}
\hline$N$ & $a_{1}^{(N)}$ & $\begin{array}{c}\text { Relative } \\
\text { error (\%) }\end{array}$ \\
\hline 1 & 1.03822 & 0.6 \\
2 & 1.03205 & 0.20 \\
3 & 1.03298 & 0.09 \\
4 & 1.03156 & 0.05 \\
5 & 1.03234 & 0.03 \\
10 & 1.03200 & 0.005 \\
\hline
\end{tabular}


Table 3

TABLE 3

\begin{tabular}{ccc}
\hline$N$ & $a_{1}^{(N)}$ & $\begin{array}{c}\text { Relative } \\
\text { error }(\%)\end{array}$ \\
\hline 1 & 1.02384 & 0.3 \\
2 & 1.01957 & 0.10 \\
3 & 1.02095 & 0.04 \\
4 & 1.02035 & 0.019 \\
5 & 1.02066 & 0.011 \\
10 & 1.02054 & 0.0015 \\
\hline
\end{tabular}


TABLE 4

\begin{tabular}{ccc}
\hline$N$ & $a_{1}^{(N)}$ & $\begin{array}{c}\text { Relative } \\
\text { error }(\%)\end{array}$ \\
\hline 1 & 0.974276 & 0.05 \\
2 & 0.974915 & 0.011 \\
3 & 0.974770 & 0.003 \\
4 & 0.974811 & 0.0011 \\
5 & 0.974796 & 0.0005 \\
10 & 0.974801 & 0.00003 \\
\hline
\end{tabular}




\section{TABLE 5}

\begin{tabular}{ccc}
\hline$N$ & $a_{1}^{(N)}$ & $\begin{array}{c}\text { Relative } \\
\text { error }(\%)\end{array}$ \\
\hline 1 & 0.956951 & 0.20 \\
2 & 0.955090 & 0.009 \\
3 & 0.955010 & 0.0004 \\
4 & 0.955006 & 0.000016 \\
5 & 0.955006 & 0.0000007 \\
10 & 0.955006 & $<10^{-10}$ \\
\hline
\end{tabular}


TABLE 6

\begin{tabular}{ccc}
\hline$N$ & $a_{1}^{(N)}$ & $\begin{array}{c}\text { Relative } \\
\text { error }(\%)\end{array}$ \\
\hline 1 & 0.936605 & 1.1 \\
2 & 0.927829 & 0.15 \\
3 & 0.926632 & 0.022 \\
4 & 0.926460 & 0.003 \\
5 & 0.926434 & 0.0005 \\
10 & 0.926430 & $<10^{-7}$ \\
\hline
\end{tabular}


TABLE 7

\begin{tabular}{ccc}
\hline$N$ & $a_{1}^{(N)}$ & $\begin{array}{c}\text { Relative } \\
\text { error }(\%)\end{array}$ \\
\hline 1 & 0.915051 & 4 \\
2 & 0.891664 & 1.5 \\
3 & 0.883503 & 0.6 \\
4 & 0.880411 & 0.23 \\
5 & 0.879193 & 0.10 \\
10 & 0.878367 & 0.0013 \\
\hline
\end{tabular}




\section{FIGURE 1}

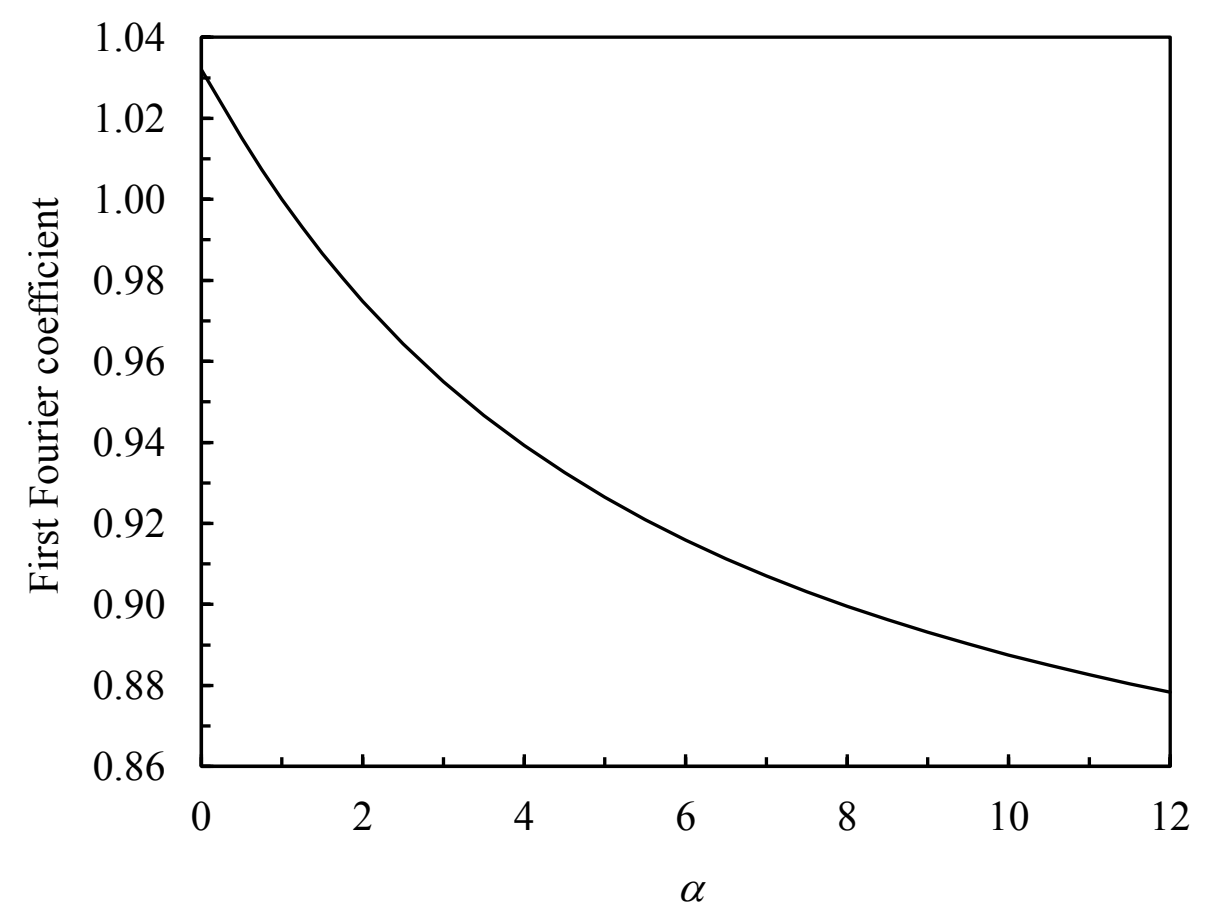




\section{FIGURE 2}

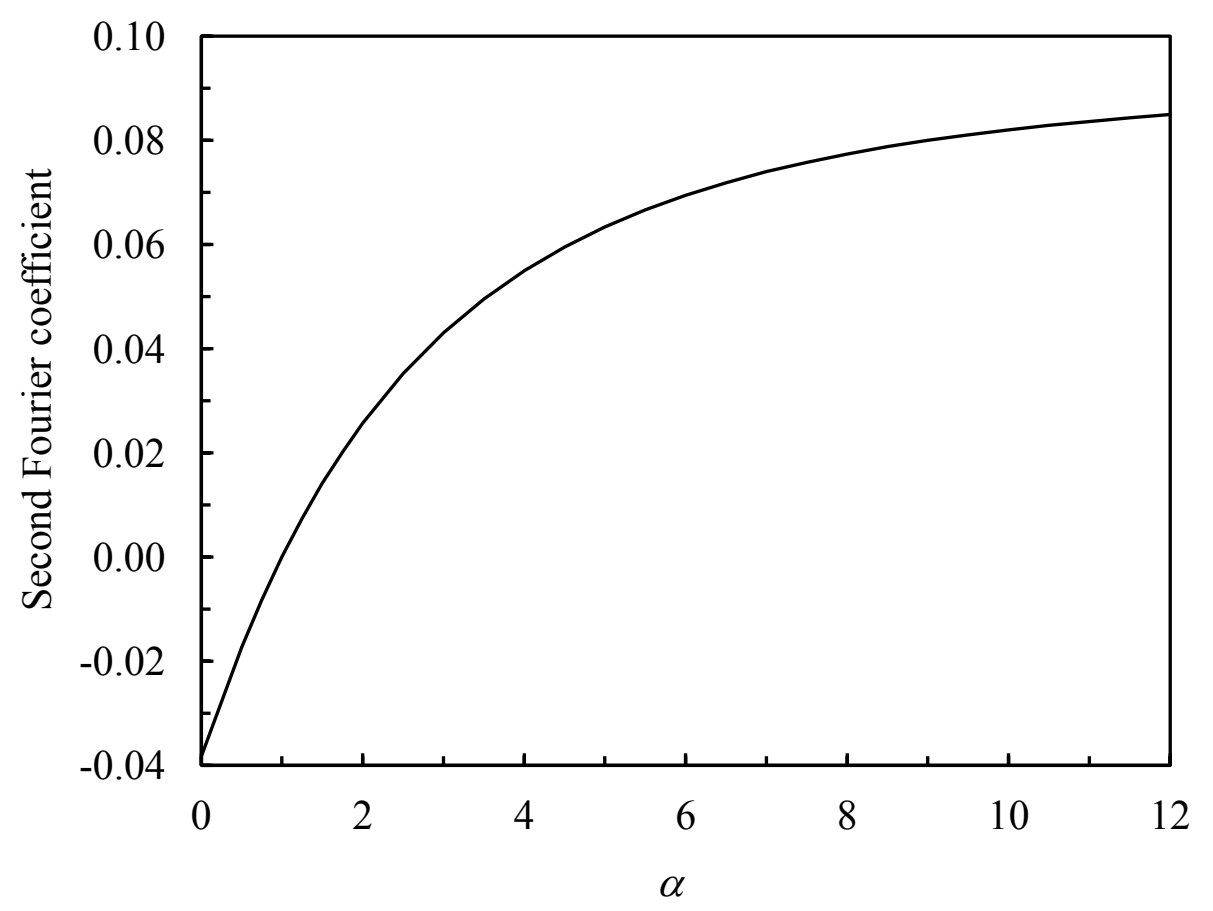




\section{FIGURE 3}

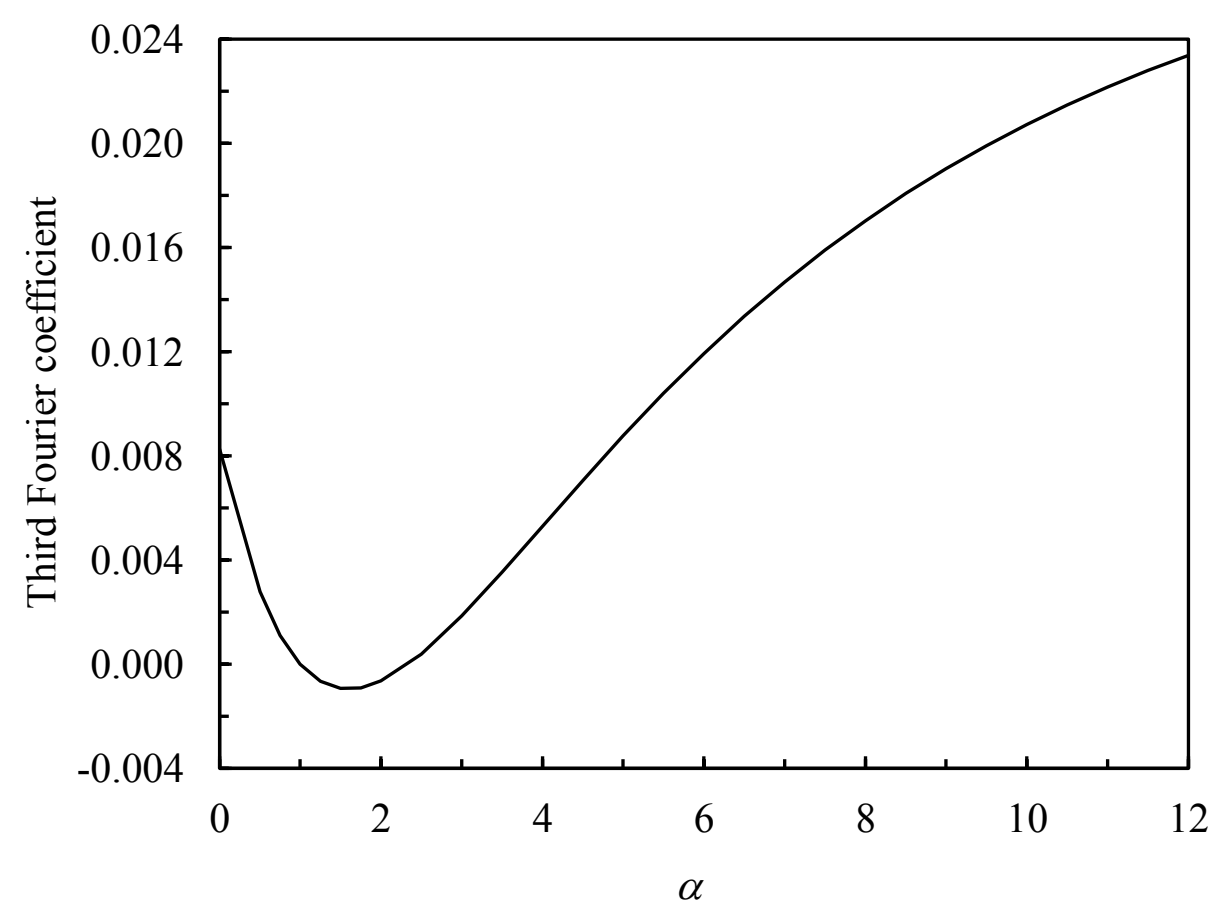




\section{FIGURE 4}

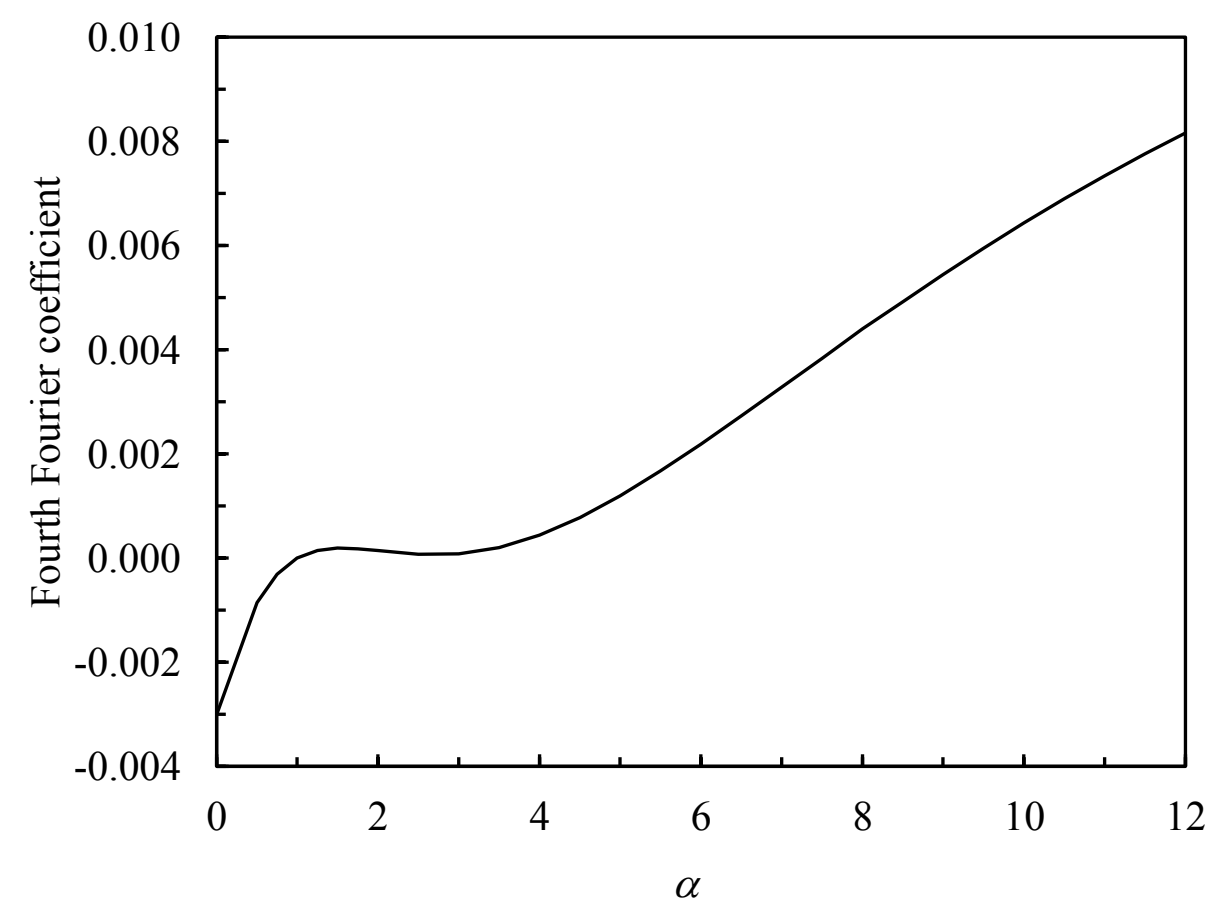




\section{FIGURE 5}

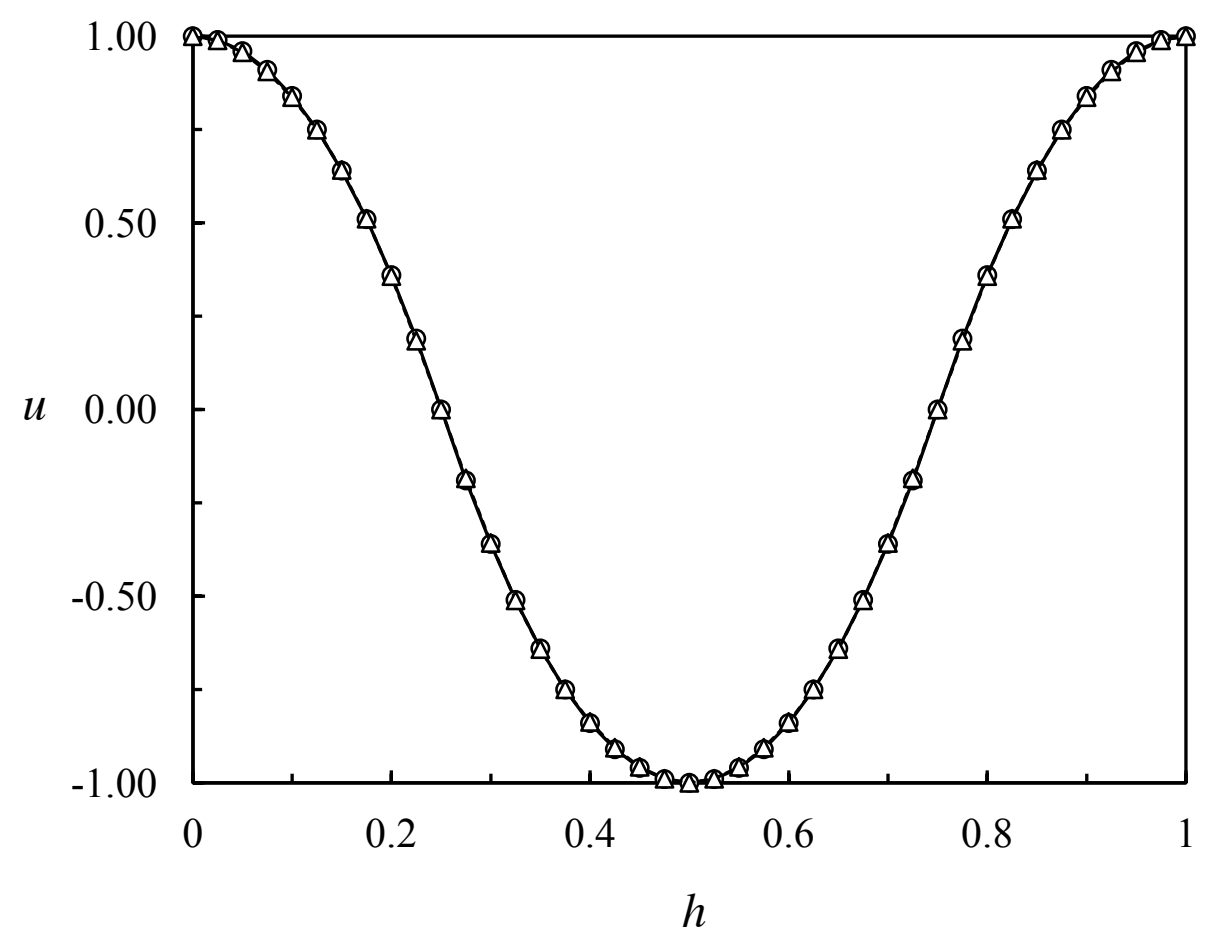




\section{FIGURE 6}

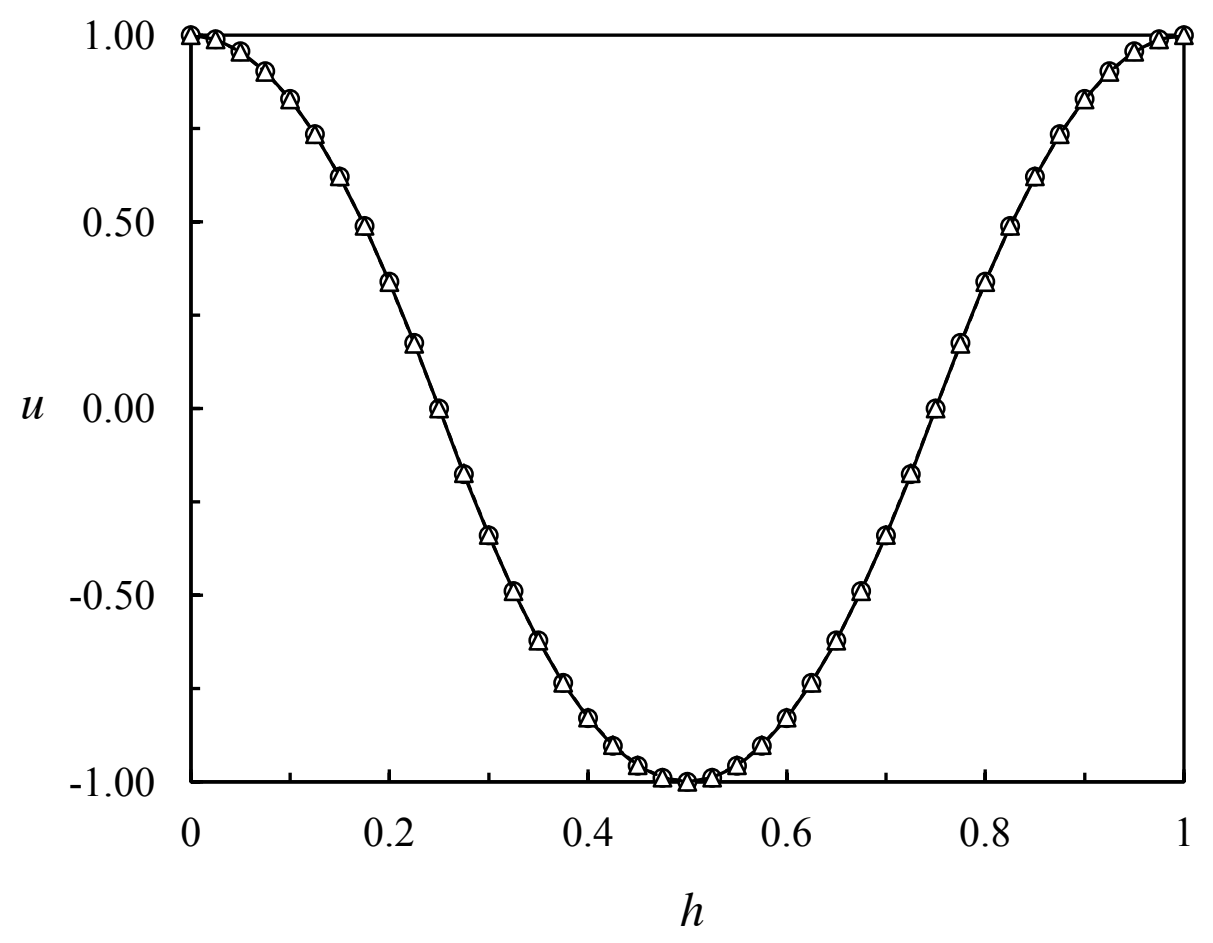




\section{FIGURE 7}

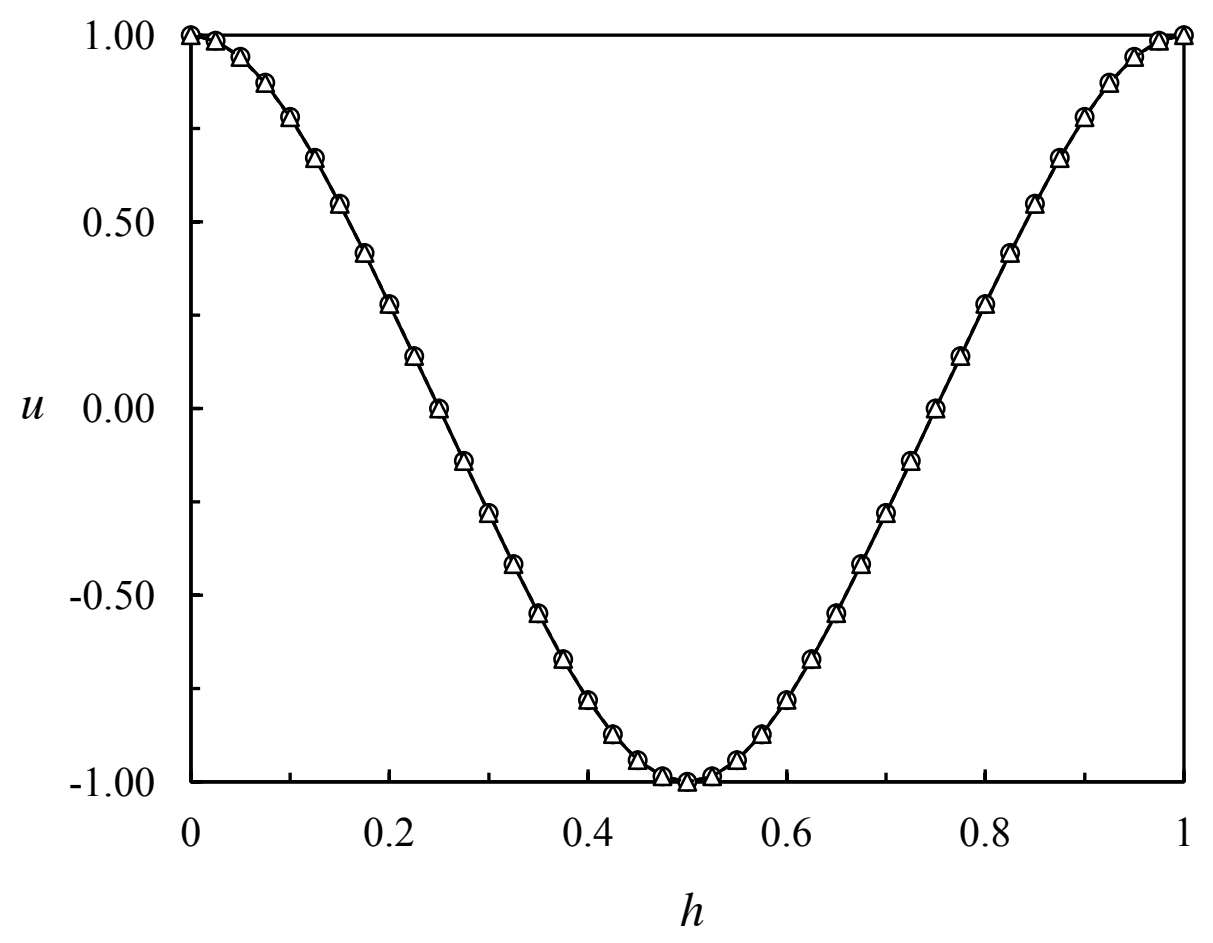




\section{FIGURE 8}

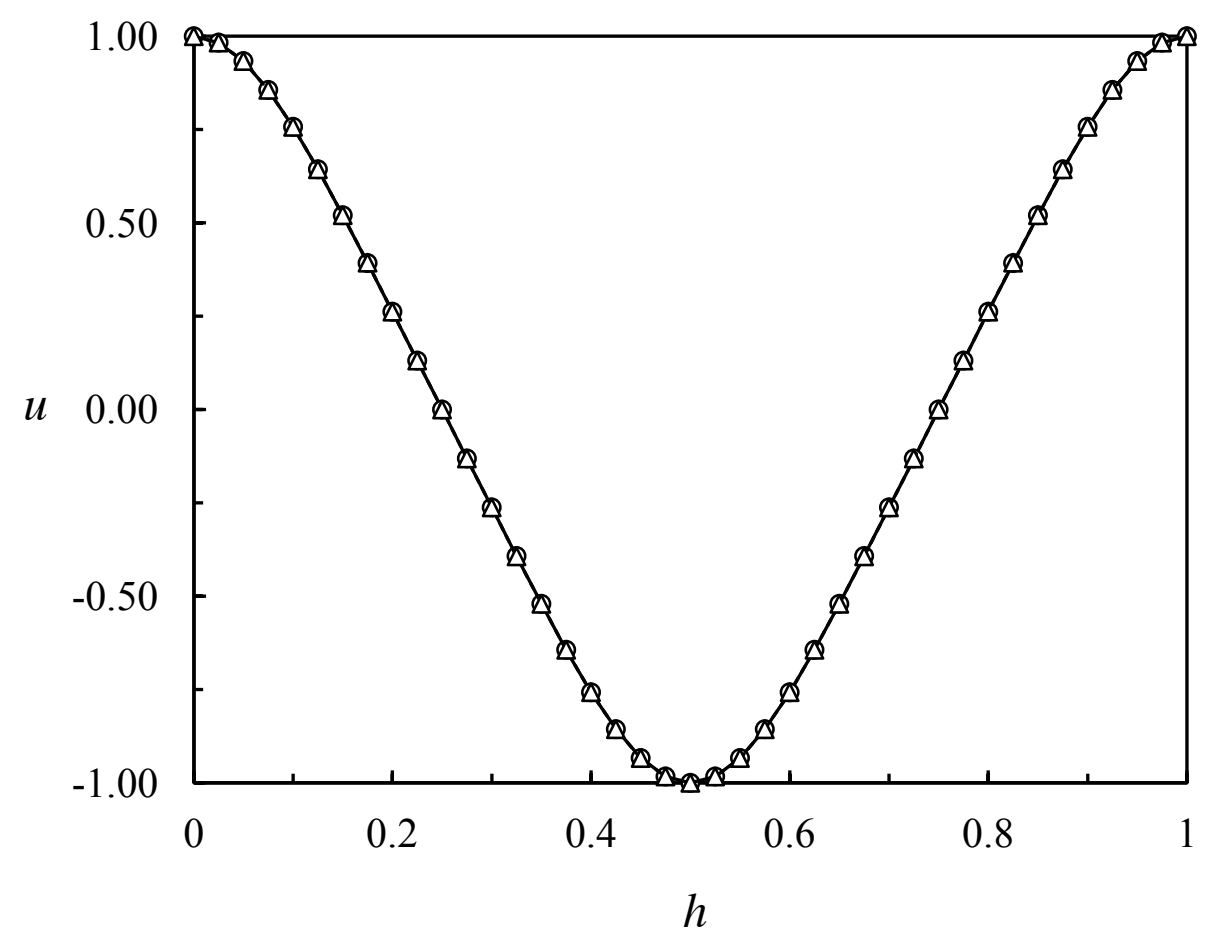




\section{FIGURE 9}

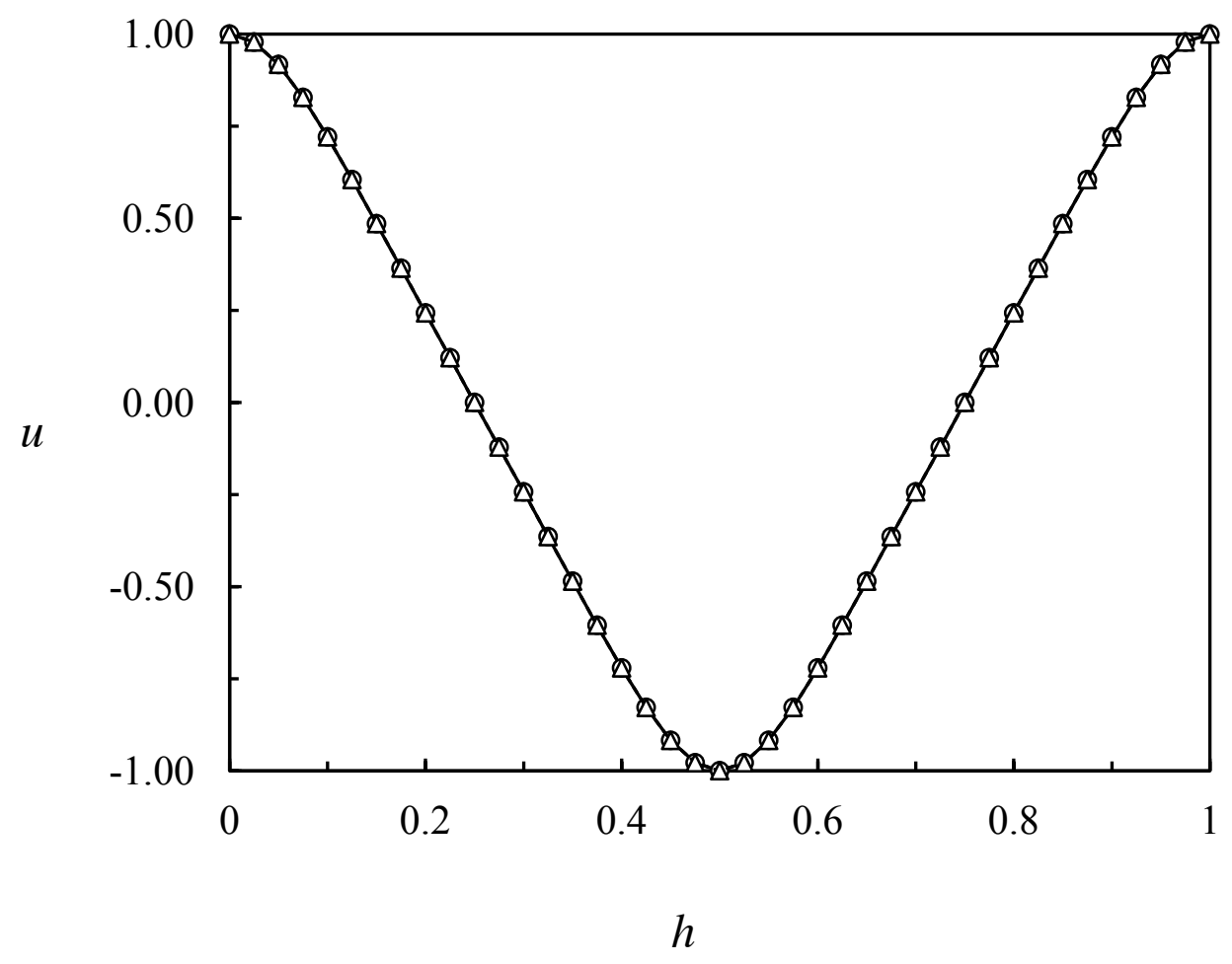




\section{FIGURE 10}

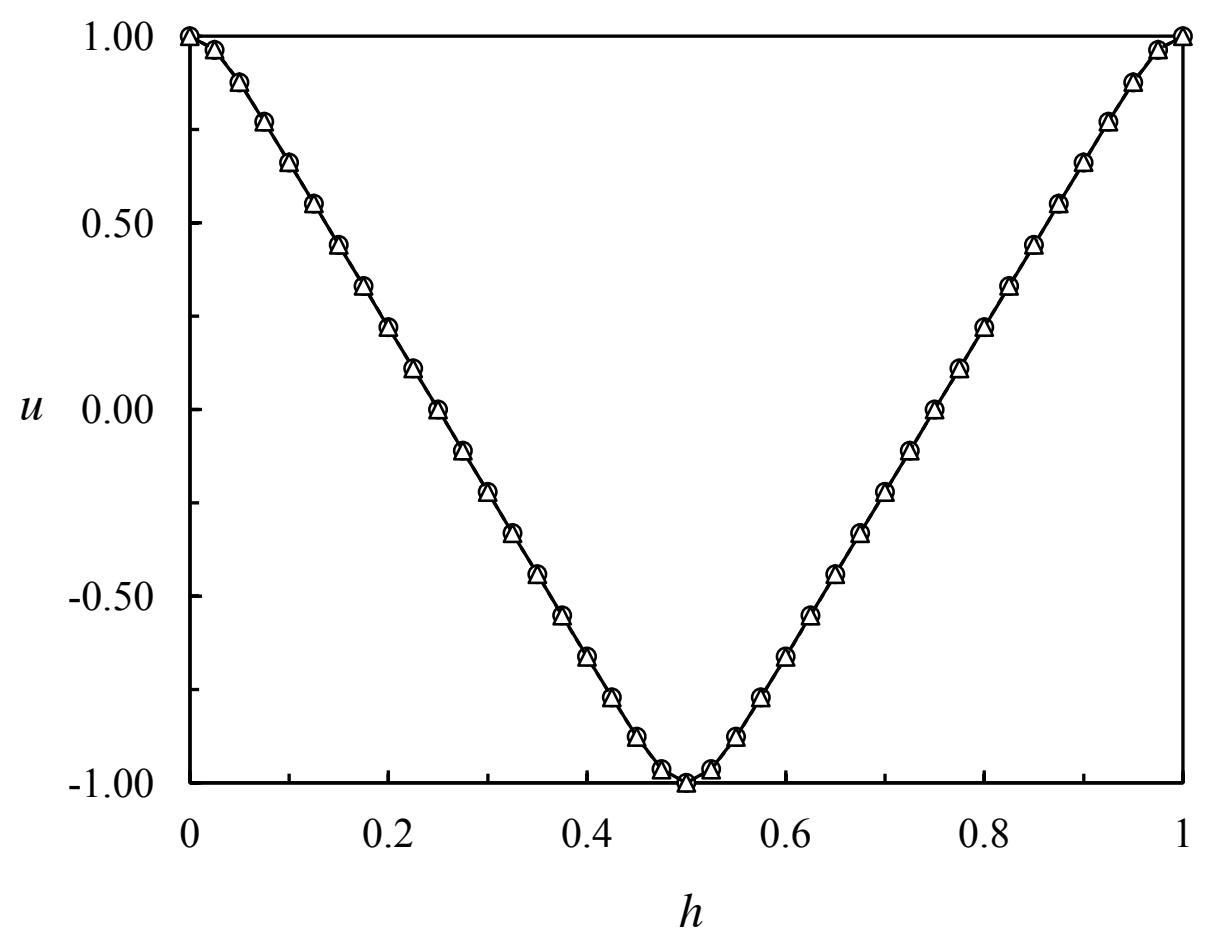




\section{FIGURE 11}

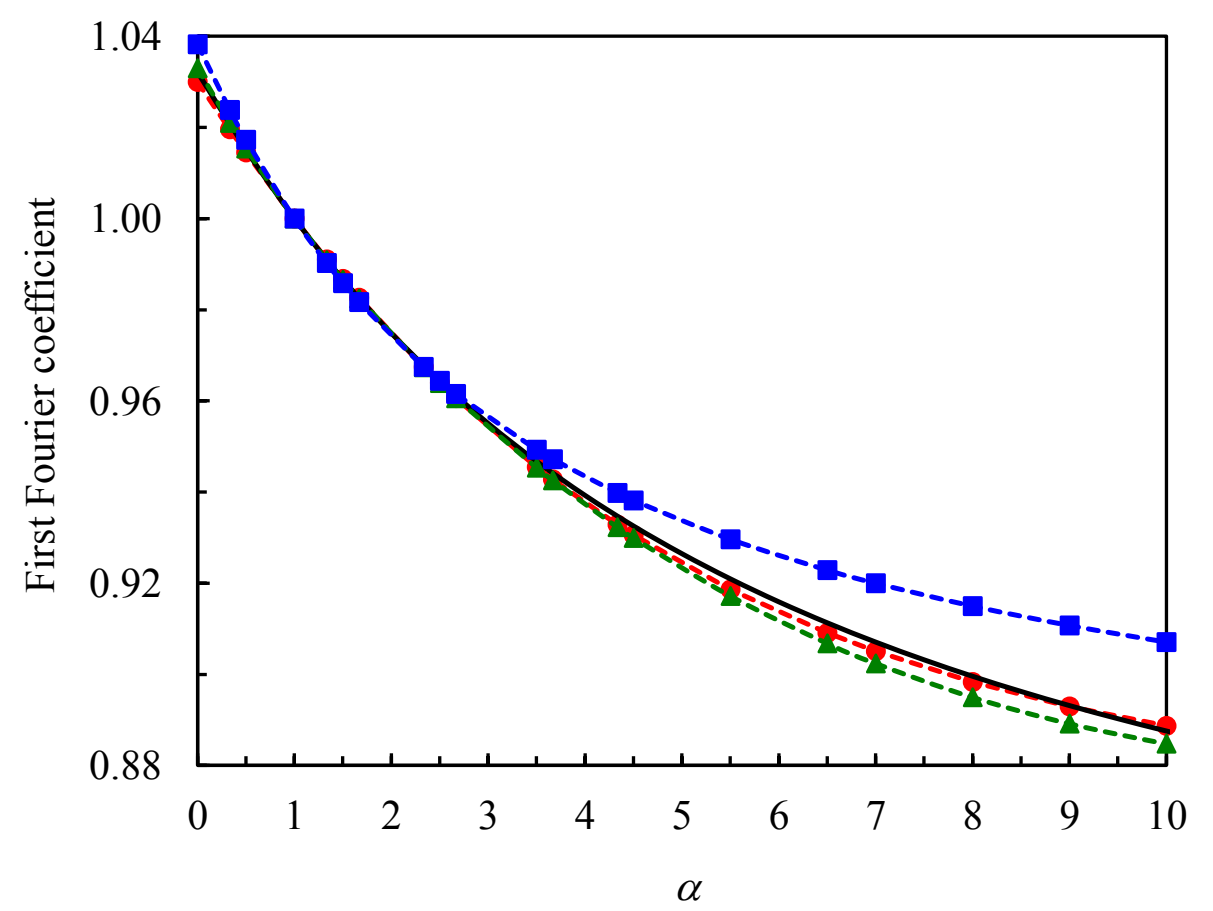




\section{FIGURE 12}

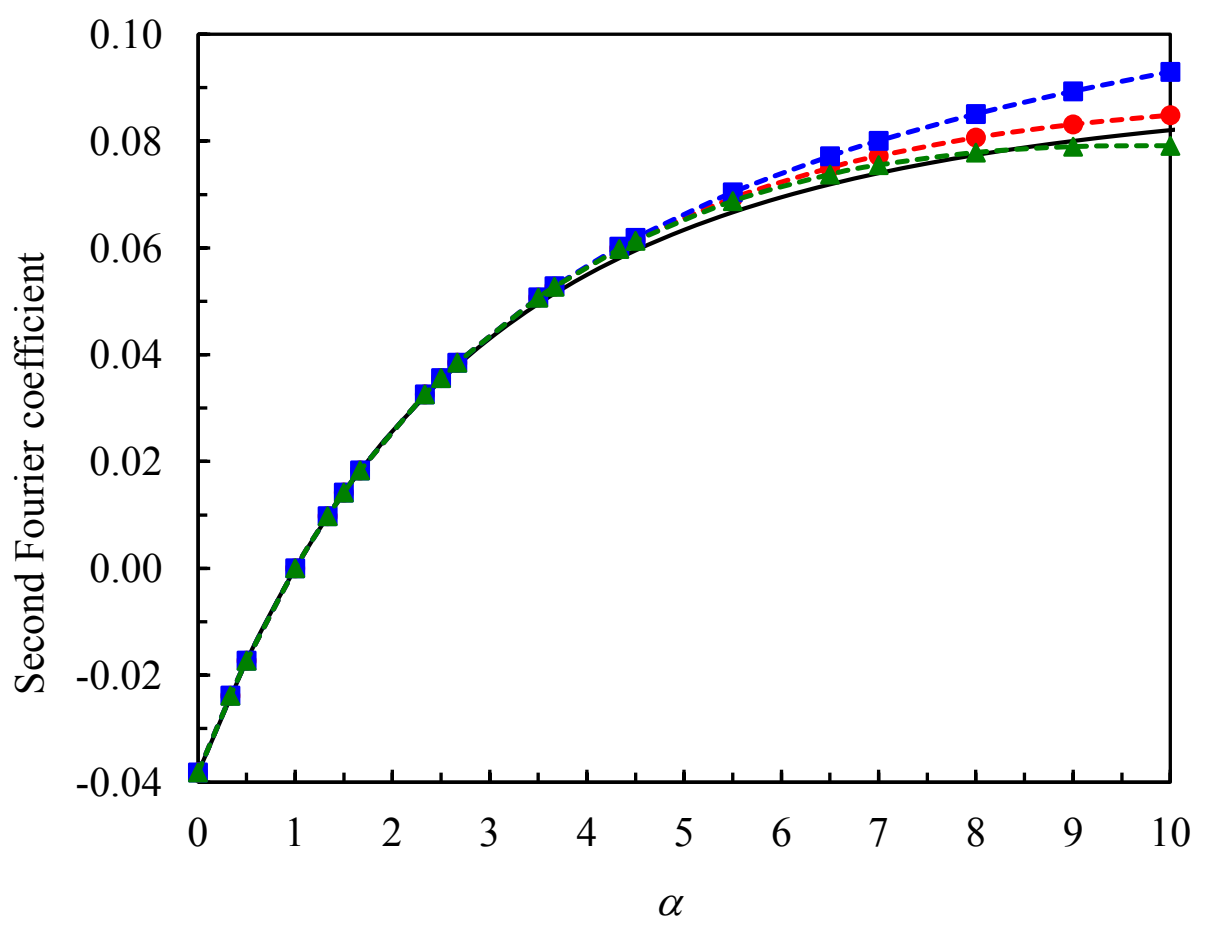




\section{FIGURE 13}

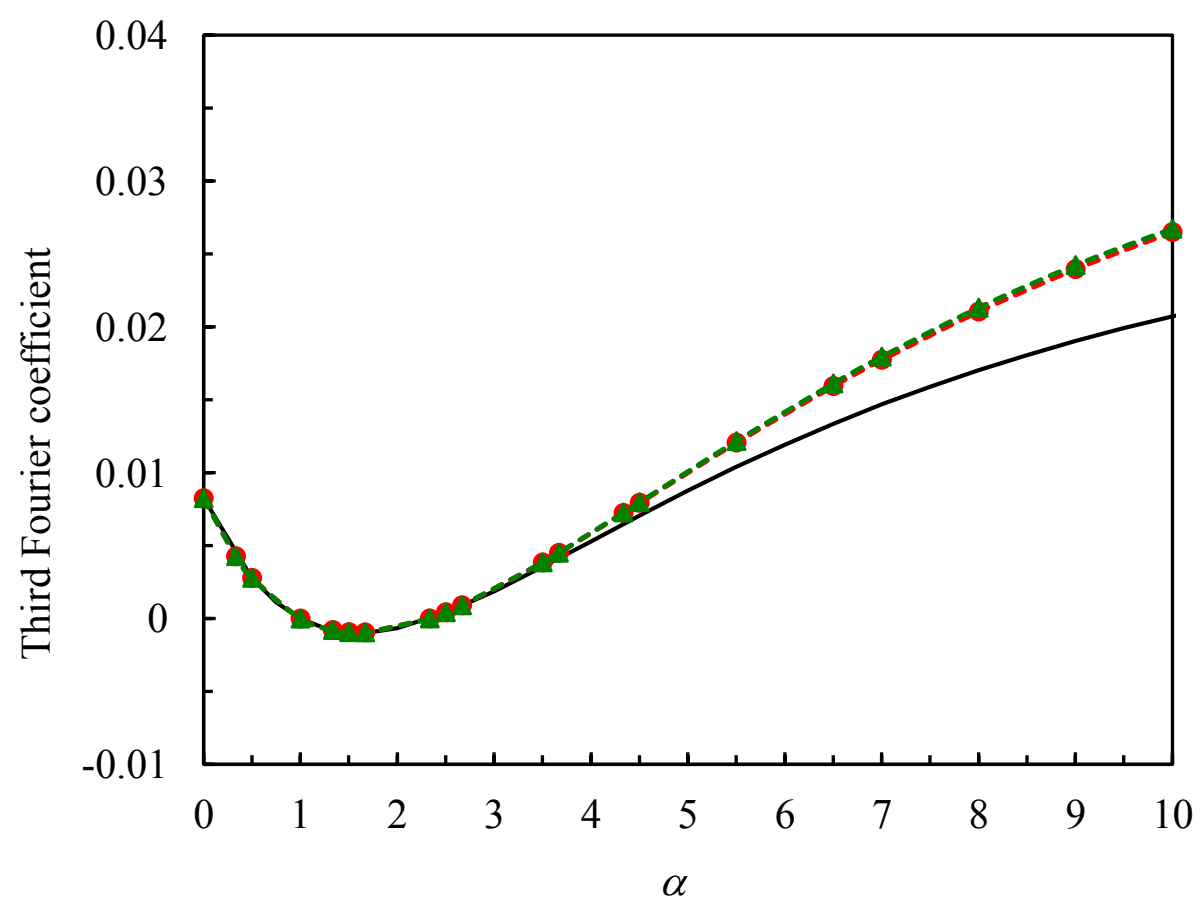




\section{FIGURE 14}

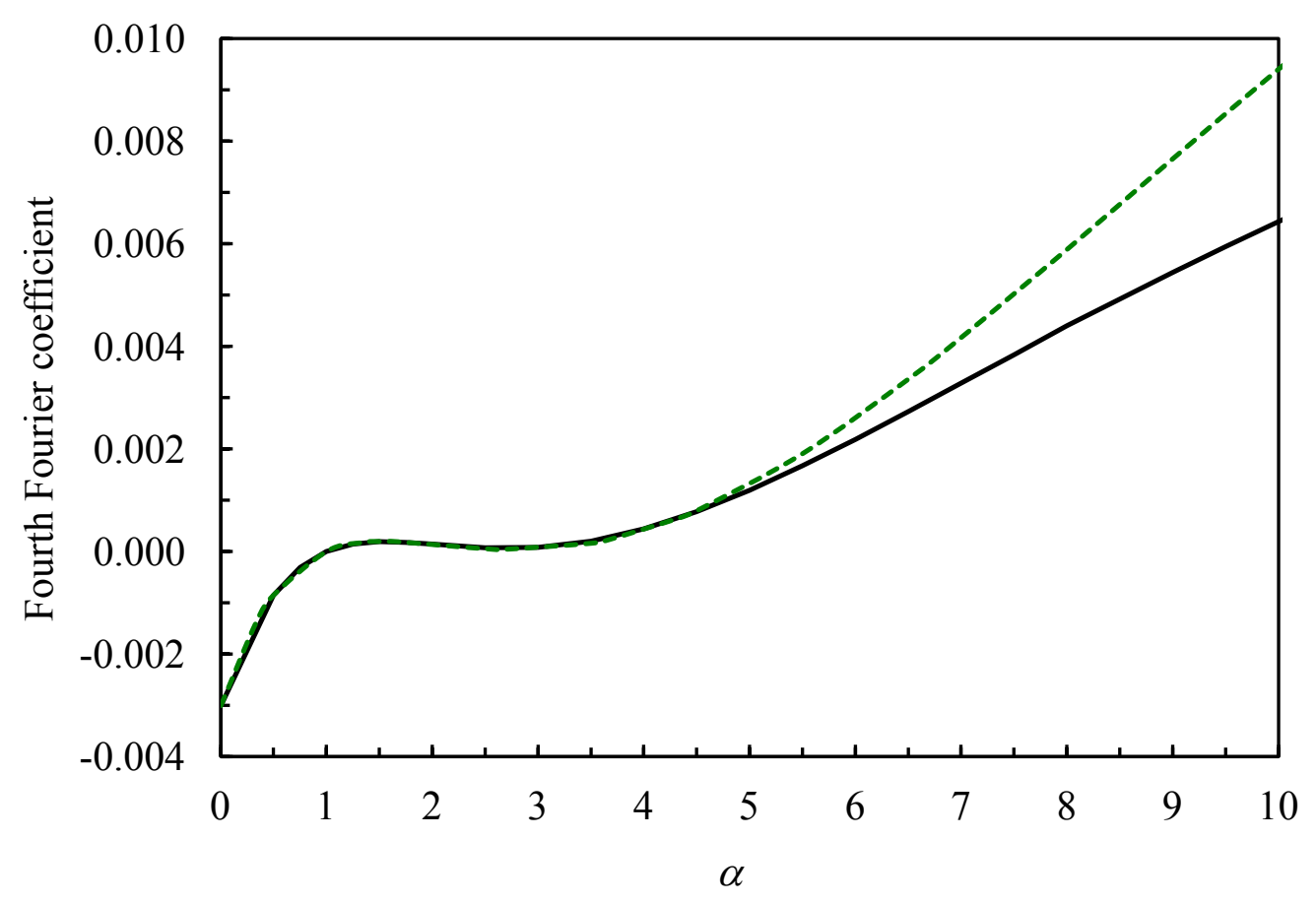

